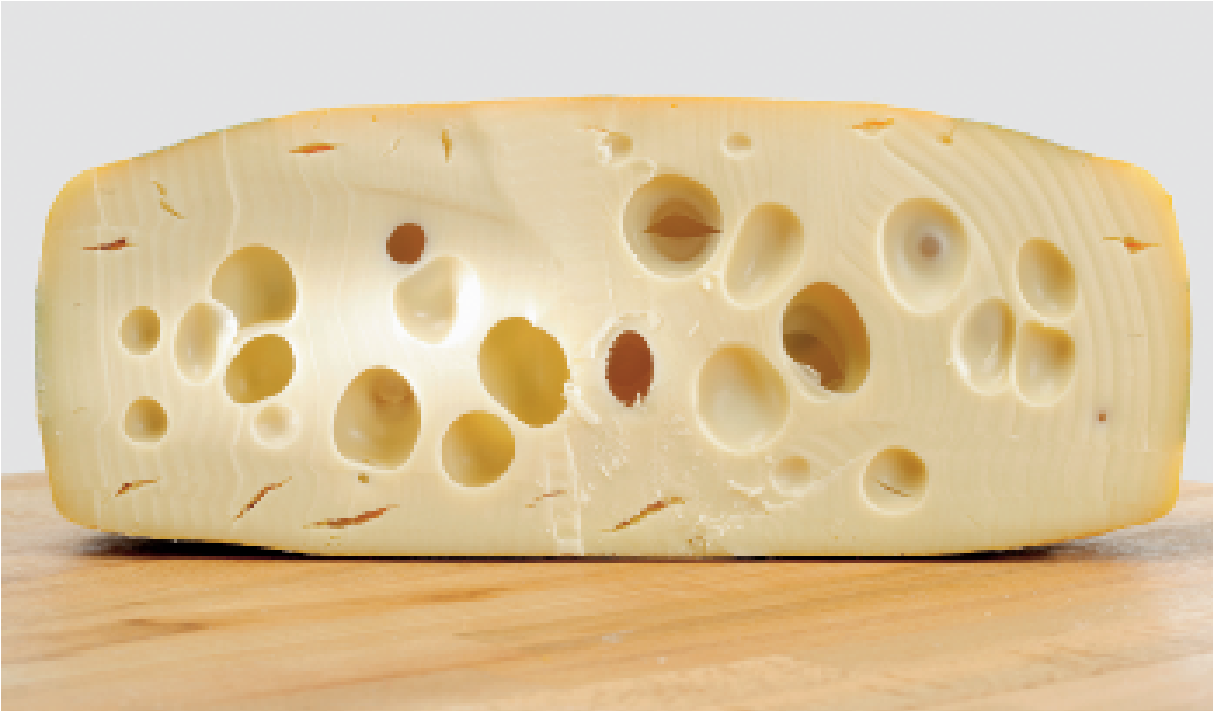

Datenschlupflöcher müssen geschlossen werden, fordert Ö̈̈K-Präsident Reiner Brettenthaler.

\section{Löchriger Datenschutz}

Mit scharfer Kritik hat sich die Österreichische Ärztekammer (ÖÄK) gegen die Praxis des Arbeitsmarktservices Oberösterreich (AMS) gewendet, sensible Gesundheitsdaten von Arbeitslosen an künftige Arbeitgeber weiterzuleiten. «Als Vertreter und Fürsprecher der Patienten in diesem Land protestieren wir auf das schärfste gegen diesen Usus, der medizinisch kontraproduktiv ist, das Vertrauen der Patienten in den ärztlichen Heilberuf untergräbt und die Chancen von Patienten, wieder im Arbeitsleben Fuss zu fassen, schmälert», erklärte ÖÄK-Präsident Reiner Brettenthaler. Der Gesetzgeber sei aufgerufen, «dieses Datenschlupfloch umgehend zu schliessen und den Schutzrechten der Patienten bei der Arbeitsvermittlung jenen Stellenwert einzuräumen, der ihnen gebührt», forderte Brettenthaler. Es könne nicht angehen, dass eine schwammige rechtliche Generalklausel die Sammlung und Weitergabe sensibler Gesundheitsdaten durch Nichtmediziner an nichtmedizinische Stellen ermögliche. «Werden potentielle Arbeitgeber demnächst auch Gentests oder psychologische Gutachten von ihren Bewerbern einfordern? Wo ist da der Unterschied?» fragte Brettenthaler. Nicht medizinisch ausgebildete Sachbearbeiter seien nicht kompetent, medizinische Daten und ihre Relevanz zu beurteilen, kritisierte der Ärztechef weiter. Brettenthaler sieht die grundsätzliche Skepsis der Ärzteschaft gegenüber der neuen elektronischen Gesundheitsakte (ELGA) durch die «humanitär durch nichts zu rechtfertigende» Praxis des AMS bestätigt. Bei der ELGA sollen künftig die Gesundheitsdaten der Österreicherinnen und Österreicher elektronisch gespeichert und zugänglich gemacht werden. «Wir sind keine Technologieverweigerer und auch nicht grundsätzlich gegen Projekte dieser Art. Aber Fälle wie diese zeigen, dass Daten in die Hände der Ärzte gehören und dort bleiben müssen, damit sie nicht an alle möglichen Stellen weitergeleitet werden. Ärzte müssen beim Vermittlungsvorgang jene Schaltstellen bleiben, ohne die nichts geht. Und Datenweitergabe darf nur nach ausdrücklicher individueller Aufklärung und Zustimmung des Patienten geschehen», schloss Brettenthaler.

(Ö̈̈K)

\section{Arztflucht in reichere Stadtteile}

In Hamburg verlassen immer mehr Ärzte ihre Praxis in den sozial schwachen Stadtteilen und wandern in die reicheren Gegenden ab. Der Grund: ein Mangel an Privatpatienten, die für das wirtschaftliche Auskommen der Praxis zwingend erforderlich sind. In den sozial schwachen Stadtteilen vieler Grossstädte fehlen die Mediziner, wie in den Hamburger Bezirken Wilhelmsburg, Veddel oder Billstedt. «Schuld ist das hohe Patientenaufkommen bei gleichzeitig geringen Budgets», berichtet das «Hamburger Abendblatt». «Um kostendeckend zu arbeiten, müsste jeder zehnte Patient privat versichert sein», erläuterte Dr. Michael Späth, Vorsitzender der Vertreterversammlung der KV Hamburg, dem Blatt. 90 Prozent der Patienten etwa in Wilhelms- burg seien jedoch bei der AOK versichert, die eine vergleichsweise geringe Kopfpauschale für ihre Mitglieder zahle. Lediglich ein bis zwei Prozent seien privat versichert. Um überhaupt noch überleben zu können, wandern daher viele Ärzte von armen in reiche Stadtteile ab. «Die Reaktion ist verständlich. Wieso sollte ein junger Arzt das Risiko auf sich nehmen und seine berufliche Existenz riskieren, um in Wilhelmsburg zu arbeiten», kommentierte die gesundheitspolitische Sprecherin der GAL-Bürgerschaftsfraktion, Katja Husen. Die Krankenkassen zahlten zu wenig für die Versorgung und Privatpatienten gebe es kaum.

(Kassenarzt)

\section{France: carte d'assurance- maladie Vitale 2}

Xavier Bertrand, ministre de la Santé et des solidarités, rappelle que la carte Vitale 2 est un outil important d'amélioration de notre système de santé. Prévue dans le cadre de la réforme de l'assurance maladie, la nouvelle carte Vitale 2 permettra notamment l'inscription, en plus des données administratives, des données personnelles relatives à: - la désignation du médecin traitant; - la personne à prévenir en cas d'urgence; - la mention que l'assuré a bien bénéficié d'une information sur le don d'organe et sur la manière de faire connaître sa position; - la protection maladie complémentaire. Comportant obligatoirement la photographie de l'assuré, elle est dotée d'une technologie plus sécurisée, de capacité d'authentification et de signature électronique. Enfin, la diffusion de la carte Vitale 2 fait l'objet d'une gestion et d'un contrôle renforcés qui garantiront l'existence d'une seule carte par bénéficiaire et préviendront les risques de fraudes. La publication du décret va permettre le lancement des opérations de fabrication des cartes par les caisses d'assurance maladie. Le déploiement de la carte Vitale 2 débutera en Bretagne à partir du mois prochain: en fonction du rythme de retour des formulaires de collecte des photographies, les premières cartes délivrées aux assurés bretons seront disponibles à la mi-mars. Les nouvelles cartes Vitale 2 seront d'abord distribuées aux assurés de plus de 16 ans qui se voient délivrer leur première carte personnelle, ainsi qu'aux assurés dont les cartes doivent être remplacées. Le déploiement sera ensuite généralisé à l'ensemble du territoire durant l'année 2007. Le renouvellement de l'ensemble des cartes des assurés sera réalisé en 4 ans et devrait s'achever en 2010.

(Communiqué) 